\title{
Single Nucleotide Polymorphism Detection Using Au-Decorated Single-Walled Carbon Nanotube Field Effect Transistors
}

\author{
Keum-Ju Lee, ${ }^{1}$ Hye-Mi So, ${ }^{1}$ Byoung-Kye Kim, ${ }^{1,2}$ Do Won Kim, ${ }^{3}$ Jee-Hwan Jang, ${ }^{3,4}$ \\ Ki-Jeong Kong, ${ }^{1}$ Hyunju Chang, ${ }^{1}$ and Jeong-O Lee ${ }^{1}$ \\ ${ }^{1}$ NanoBio Fusion Research Center, Korea Research Institute of Chemical Technology, Daejeon 305-343, Republic of Korea \\ ${ }^{2}$ Regional Innovation Agency, Jeonbuk Technopark, Jeonju 561-844, Republic of Korea \\ ${ }^{3}$ Panagene Inc., Daejeon 305-510, Republic of Korea \\ ${ }^{4}$ Ucaretron Inc., Dongiltechno Building C, Anyang 431-716, Republic of Korea \\ Correspondence should be addressed to Jeong-O Lee, jolee@krict.re.kr
}

Received 14 June 2010; Revised 3 September 2010; Accepted 10 September 2010

Academic Editor: Jianyu Huang

Copyright ( $\odot 2011$ Keum-Ju Lee et al. This is an open access article distributed under the Creative Commons Attribution License, which permits unrestricted use, distribution, and reproduction in any medium, provided the original work is properly cited.

\begin{abstract}
We demonstrate that Au-cluster-decorated single-walled carbon nanotubes (SWNTs) may be used to discriminate single nucleotide polymorphism (SNP). Nanoscale Au clusters were formed on the side walls of carbon nanotubes in a transistor geometry using electrochemical deposition. The effect of Au cluster decoration appeared as hole doping when electrical transport characteristics were examined. Thiolated single-stranded probe peptide nucleic acid (PNA) was successfully immobilized on Au clusters decorating single-walled carbon nanotube field-effect transistors (SWNT-FETs), resulting in a conductance decrease that could be explained by a decrease in Au work function upon adsorption of thiolated PNA. Although a target single-stranded DNA (ssDNA) with a single mismatch did not cause any change in electrical conductance, a clear decrease in conductance was observed with matched ssDNA, thereby showing the possibility of SNP (single nucleotide polymorphism) detection using Au-cluster-decorated SWNT-FETs. However, a power to discriminate SNP target is lost in high ionic environment. We can conclude that observed SNP discrimination in low ionic environment is due to the hampered binding of SNP target on nanoscale surfaces in low ionic conditions.
\end{abstract}

\section{Introduction}

The ability to detect small sequence differences in DNA molecules is very important in molecular biology, especially in the context of personalized diagnostics and therapy [1]. PCR combined with fluorescence detection is the most widely used and commercialized technique to date; however, this method requires expensive, bulky instruments, which has hindered the popularization of DNA-based diagnostics. There is a great demand for cost-effective, miniaturized DNA diagnostic sensors. Electronic detection platforms such as electrochemical or field-effect transistor sensors may be ideal, because electronic sensors do not require expensive instrumentation, and large-scale production is easy using microfabrication technology. Extensive efforts have been made to develop such DNA sensors. Two distinct approaches have been taken. In one approach, oligomers are used to mea- sure hybridization of target DNA, while in the other, mutations are probed using enzymes recognizing single nucleotide polymorphisms (SNPs), deletions, translocations, or inversions $[2,3]$. The first method (hybridization detection) using hairpin probe DNAs with redox or enzyme tags has been quite effective in development of electrochemical sensors [46], and most reported DNA sensors of the FET type use this method. For example, Hahm and Lieber reported realtime detection of DNA using peptide nucleic acid (PNA-) modified Si nanowire devices [7], and DNA sensors based on single-walled carbon nanotube field effect transistors (SWNT-FETs) have also been reported [8]. Silicon nanowire devices have the advantage of well-established surface chemistry techniques borrowed from the silicon industry. However, immobilization of probe molecules onto carbon nanotubes remains challenging. If probe molecules are covalently bound to the nanotubes, the carbon bonds will be broken, 
thus destroying the excellent electrical transport characteristics of individual nanotubes. Thus, biosensors using SWNTFETs bearing noncovalently attached linkers such as pyrene or CDI-Tween 20 have been developed $[9,10]$. However, the successful use of noncovalent binding linkers for hydrophilic molecules such as DNA remains to be demonstrated.

Recently, Star et al. showed label-free detection of DNA hybridization using nanotube network transistors [8]. In their example, probe single-stranded DNAs (ssDNAs) were nonspecifically adsorbed onto carbon nanotubes by $\pi$ stacking between DNA bases and the sidewalls of SWNTs. Such nonspecifically adsorbed molecules cannot easily bind with complementary strands; it has been shown that ssDNA forms a complex with an SWNT by wrapping around the SWNT $[11,12]$. Jeng et al. showed that although target DNA could hybridize with probe ssDNA on SWNTs, the process was relatively slow ( 13 hours at $25^{\circ} \mathrm{C}$, compared to less than 10 minutes for free DNA hybridization) [13]. Therefore, an immobilization method better than that offered by simple nonspecific binding is required. To that end, we developed Au-nanoparticle-decorated SWNT-FETs as sensor platforms for label-free detection of DNA hybridization. Thiolated DNA capture probes bind to the $\mathrm{Au}$ nanoparticles on SWNTs, but the nanoparticles do not disturb the excellent electrical properties of SWNTs. Thus, we may covalently attach DNA capture probes and measure electrical responses upon hybridization. Metallic nanoparticles may offer a solid support for molecular recognition elements, and incorporating metal nanoparticles into SWNT sensors has been shown to effectively enhance the sensitivity [14-16].

\section{Results and Discussion}

The fabrication of devices using patterned growth techniques and the fabrication details are described in the Method section. Figure 1(a) shows an atomic force micrograph of a device decorated with Au nanoparticles. Devices with only one or two nanotubes between electrodes were used for all experiments. Figure S1(b) (in Supplementary Material available online at doi:10.1155/2011/105138) shows the change in $I-V_{g}$ characteristics upon Au decoration by electrochemical deposition. Several different approaches are available for $\mathrm{Au}$ nanoparticle decoration of SWNTs. For example, SWNTs may be decorated by chemical modification [17]. However, a "physical" decoration method is preferable. We have explored a simple evaporation method as well as spontaneous reduction [18]. With physical evaporation, nonspecific probe immobilization is problematic, and it is difficult to control Au nanoparticle size with spontaneous reduction. Therefore, we used electrochemical deposition [19-21].

Unlike the electroless deposition method, electrodeposition of $\mathrm{Au}$ nanoparticles does not require oxidative consumption of nanotubes. Therefore, the observed hole-doping effect may be explained by the work function difference between the metal and the SWNT. When the high-workfunction metal $\mathrm{Au}(\approx 5.2 \mathrm{eV})$ is brought into contact with an SWNT, electrons will be transferred from the SWNT to the Au by virtue of the work function difference [22]. One of the advantages with metal decoration on SWNTs is that the reactions can be observed with optical microscope. A single SWNT decorated with Au nanoparticles larger than $50 \mathrm{~nm}$ can be directly imaged with optical microscopes, and dye molecules immobilized on Au nanoparticles can be imaged with confocal laser microscope. As shown in Figure 1(b) and Figure S2 in Supplementary Material, strong fluorescence signals observed upon Cy-3-labeled PNA binding. Usually, fluorescence signals from metal surfaces are low because of surface energy transfer [23]. The enhanced fluorescence that we observed presumably arose from coupling between $\mathrm{Au}$ nanoparticles [24].

As a probe, we used PNA rather than DNA. PNA is an artificially synthesized polymer similar to DNA and is used in both biological research and medical treatment [25]. Whereas DNA has a negatively charged phosphate backbone, the PNA backbone is composed of repeating N(2-aminoethyl)-glycine units linked by peptide bonds. PNA has remarkable thermal, chemical, and enzymatic stability, and unique ionic strength effects relative to the natural analog, DNA. Hybridization of ssDNA with complementary target DNA can be performed only in an ionic environment that stabilizes the negatively charged backbones, because of Coulombic repulsion between negatively charged molecules. However, as PNA has a neutral peptide backbone, constraints on hybridization conditions are less severe than with DNADNA hybridization [26].

PNA probe sequences can be covalently immobilized to $\mathrm{Au}$ nanoparticles decorated on SWNT-FETs using Au-S binding. In our experiment, 5' -HS-GAC ATT ACT CAC CCG-Cy3-3', with the $5^{\prime}$ end terminated by a thiol, was used as a probe PNA, whereas $5^{\prime}$-CGG GTG AGT AAT GTC$3^{\prime}$ was the target. The DNA sequences were chosen from an Escherichia coli library and synthesized by Bioneer Inc (Daejeon, Korea). Probe PNA solution was deposited on SWNT-FETs decorated with Au-nanoparticles and allowed to react for 6 hours in a humidified chamber at room temperature (Figure S2 in Supplementary Material). To confirm successful immobilization, Cy-3-labeled probe PNA was employed. Figures 1(a) and 1(b) show an AFM image and a confocal microscope image of an Au-decorated SWNTFET bearing Cy-3-labeled PNA.

To measure hybridization of target DNA electronically, we first immersed the PNA-immobilized, Au-decorated SWNT-FET in DI water with an $\mathrm{Ag} / \mathrm{AgCl}$ reference electrode as a liquid gate and applied bias voltages $(100-300 \mathrm{mV})$ to the device with measurement of current in real time. Positive bias voltages were applied to enhance the hybridization of target DNA [27]. When device signals became stabilized, we added $5 \mu \mathrm{L}$ of $100 \mathrm{pmol} / \mu \mathrm{L}$ target DNA solution in DI water. Figure 2(a) shows real-time measurements from the device. A decrease in conductance was observed as soon as complementary target DNA was added. After about 20 minutes, the conductance saturated at $40 \%$ of the original value. The inset of Figure 2(a) shows an AFM image of the Au-decorated device. Figure 2(b) shows the evolution of $I-V_{g}$ characteristics upon immobilization of PNA and hybridization of DNA.

The original device had ambipolar characteristics, and, as is clear from the graph, the gate threshold voltage showed 


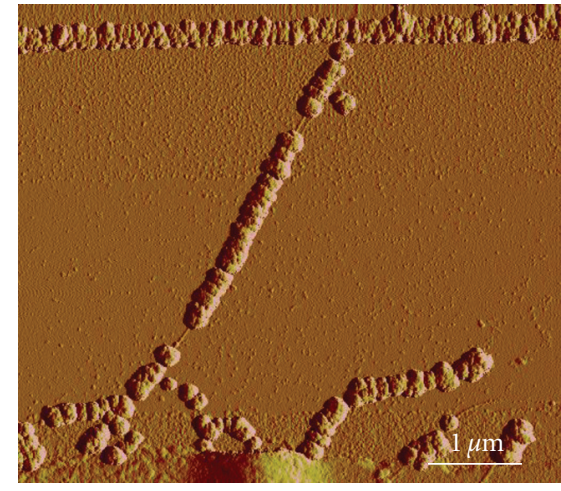

(a)

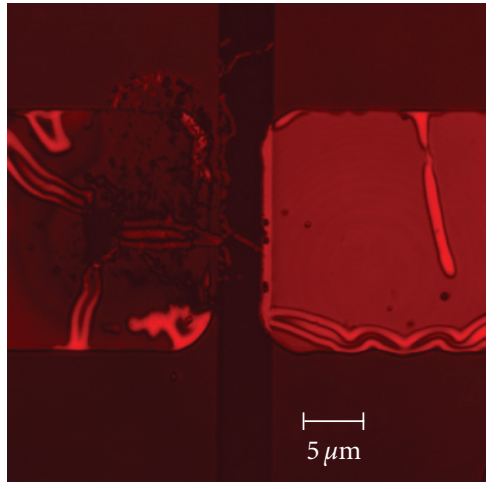

(b)

FIGURE 1: Immobilization of Cy-3-labeled probe PNA. (a) AFM image of a Au-decorated SWNT-FET. (b) Confocal micrograph of the SWNT-FET in Figure 1(a) after immobilizing Cy3-labeled probe PNA. All confocal micrographs were taken with the Zeiss LSM Exciter, with excitation at $543 \mathrm{~nm}$

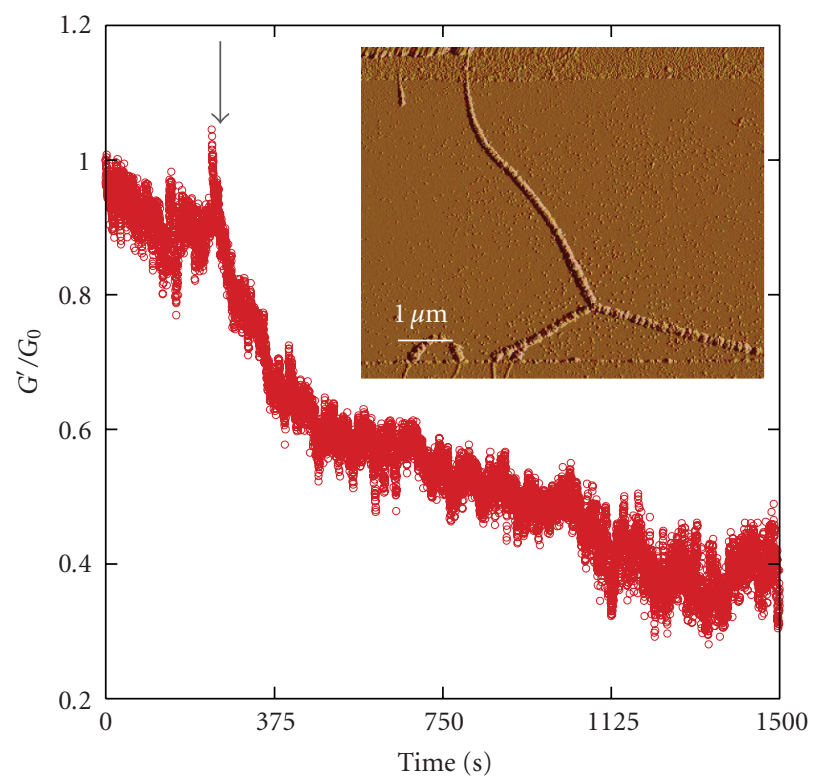

(a)

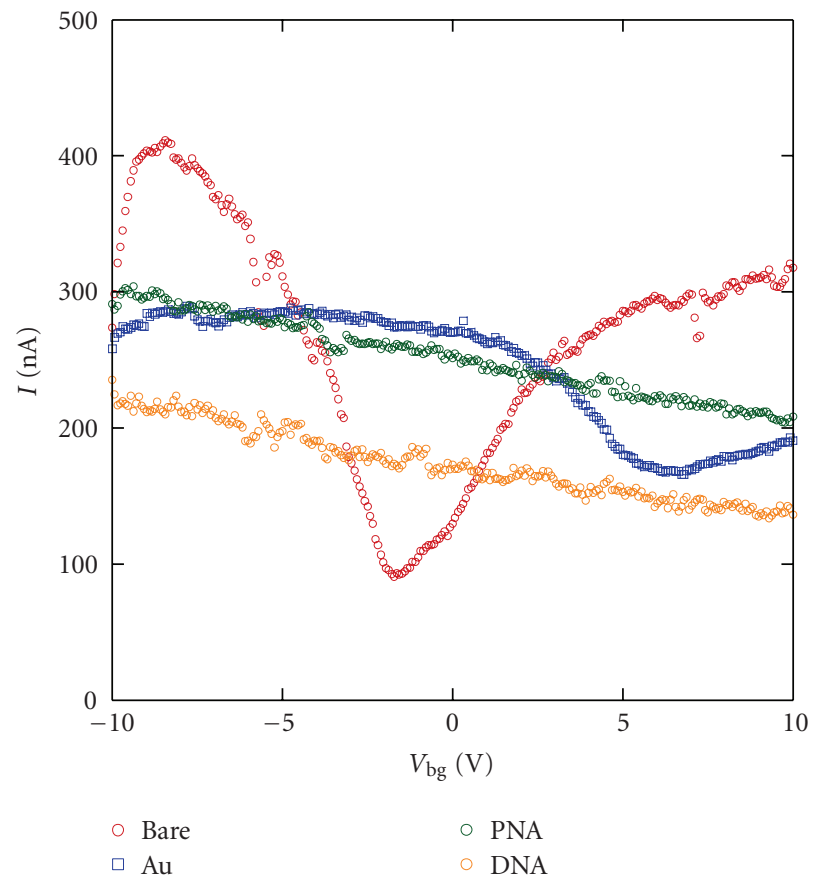

(b)

FIgURE 2: (a) The effect of hybridization on real-time conductance measured using a PNA-immobilized, Au-decorated SWNT-FET. Target DNA of a perfect match was added to the sample at the time marked with the arrow. Inset shows an AFM image of the device used for this experiment. (b) Evolution of electrical characteristics upon hybridization at $V_{\mathrm{sd}}=100 \mathrm{mV}$.

a further positive shift after Au decoration, which may be explained by the hole-doping effect of Au nanoparticles. The decrease in the on-current observed after decoration is possibly attributable to enhanced scattering at the nanotube surface [15], but the change of electrical characteristics upon Au decoration is also dependent upon decoration condition (particle size, density, etc.). Immobilization of probe PNA results in a decrease in conductance, presumably because of a reduction in the Au work function upon Au-S binding $[28,29]$. However, evolution of gate threshold is not clear in these $5 \mu \mathrm{m}$-long channel Au-decorated SWNT-FETs after PNA immobilization. We can also consider the effect of capacitance change as a dominant mechanism behind the observed conductance change. It is known that capacitance mechanism plays a major role only in the case of nearly-full covered biomolecules [30], and we expect highly packed PNA molecules on $\mathrm{Au}$ nanoparticles with 6 hours incubation. Hybridization of target DNA results in a further conductance decrease, again probably arising from a drop in the $\mathrm{Au}$ work function upon hybridization or decreased capacitance. 
Recently, lowering of the Au work function after immobilization of probe DNA and hybridization with target DNA has been observed by Kelvin probe microscopy (KPFM) [31]. Also, DNA sensors based on nanotube Schottky transistors showed decreased conductance upon hybridization related to falls in Au work function $[32,33]$.

To confirm that the observed change of conductance indeed originated from hybridization of target DNA, we fabricated Au-decorated ultralong SWNT-FETs. Ultralong SWNTs were grown on $\mathrm{SiO}_{2} / \mathrm{Si}$ substrates [34], and electrical leads were patterned using photolithography and liftoff. Immobilization of PNA and hybridization of DNA were performed as in the case of $5 \mu \mathrm{m}$-channel devices. Figure 3(a) shows a confocal microscope image of an $\mathrm{Au}-$ decorated ultralong SWNT-FET after hybridization of Cy3 labeled target DNA. Inset of Figure 3(a) is an optical micrograph of the sample, and the white arrow indicates Au-decorated SWNT. The channel length of this device is $100 \mu \mathrm{m}$, and the active channel length decreased to $80 \mu \mathrm{m}$ after electrode passivation. Note that SWNT is visible with normal optical microscope after Au decoration. As shown in the confocal micrograph of Figure 3(a), clear fluorescence signal observed from $\mathrm{Au}$ nanoparticles after hybridization of Cy-3-labeled DNA. We also confirmed that hybridization occurred mainly on Au nanoparticles by using an ultralong nanotube decorated with only a few $\mathrm{Au}$ nanoparticles (Figure S3 in Supplementary Material). Figure 3(b) shows an evolution of gate-transfer characteristics from ultralong SWNT. Again, decoration of Au nanoparticles appears as hole doping (positive shift of gate threshold voltages), and immobilization of PNA appears as decrease of conductance as well as negative shift of gate threshold voltages. Hybridization of target DNA yields further decrease of conductance. Since the active channel length is much longer in ultralong SWNTs, we may expect that pronounced doping effect (gating effect) plays a role in them. Decrease of conductance upon hybridization of matching sequence was universal for both short-channel $(5 \mu \mathrm{m})$ and long-channel $(80 \mu \mathrm{m})$ devices. In Figure S4 in Supplementary Material, we showed electronic transfer characteristics of 5 different devices [(a), (b), and (c) from short-channel devices, while (d) and (e) are from long-channel devices]. As is clear from the graphs, decoration of Au nanoparticles appears as hole doping, and immobilization of probe PNA causes decrease of conductance, as well as negative shift of gate threshold voltages. Hybridization of target DNA appears as a decrease of conductance and negative shift of gate threshold voltage in all five samples, though conductance change $\Delta I$ could vary $20 \sim 50 \%$ from different devices. To test the advantages of $\mathrm{Au}-$ decorated SWNT-FET, we performed the same experiment with nonspecifically bound probe PNA molecules. Bare SWNT-FET devices were incubated with PNA solution for 6 hours, and hybridization experiment was followed after thorough washing. However, devices with nonspecifically bound PNAs show little consistency. As shown in Figure S5 of Supplementary Material, $60 \%$ of the devices showed decrease of conductance, $30 \%$ of the devices did not show any change of conductance, and the rest showed increase of conductance. Since the nonspecific binding of probe PNA (DNA) depends

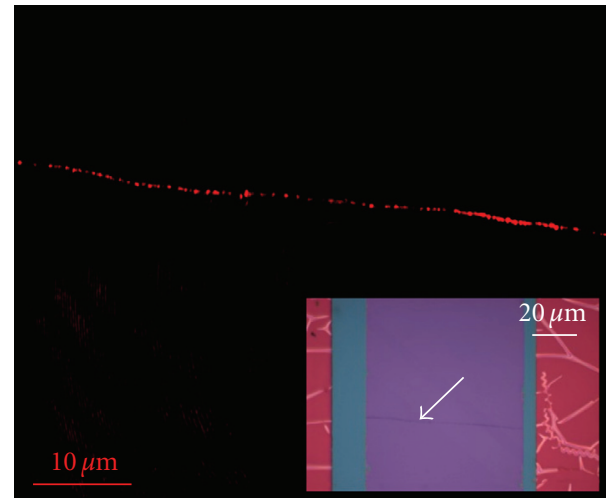

(a)

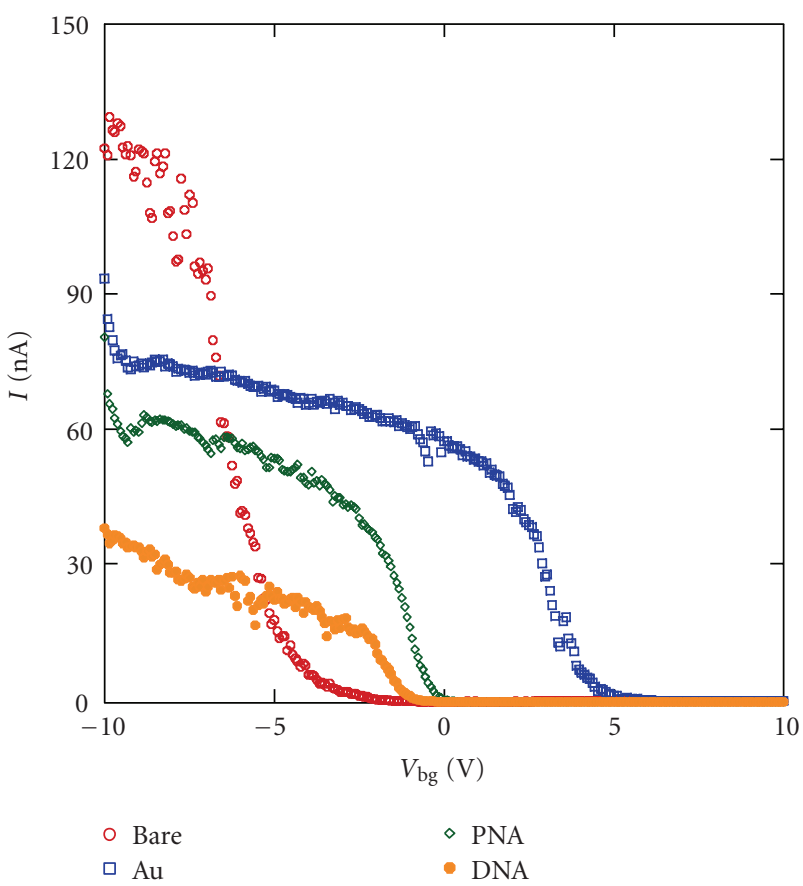

(b)

Figure 3: Hybridization of DNA on PNA-grafted, Au-decorated ultralong SWNT-FET. (a) Confocal micrograph of the Audecorated, PNA grafted ultralong SWNT after the hybridization of Cy-3 labeled target DNA. Inset shows an optical microscope image of the device, and the white arrow indicates Au-decorated ultralong SWNT. (b) Evolution of electrical characteristics upon hybridization at $V_{\text {sd }}=1 \mathrm{~V}$. Clear decrease of conductance and negative shift of gate threshold voltages observed upon hybridization.

heavily on the chirality of individual nanotubes, it may be difficult to have consistency with individual SWNT devices that have random chirality.

To determine whether the Au-decorated SWNT-FET could detect an SNP, we used 5' -CGG GTG AAT AAT GTC$3^{\prime}$ as target ssDNA. Figure 4(a) shows real-time conductance values from this experiment. In contrast to the current change seen when a perfectly matched target was added, use of target ssDNA with a single mismatch did not alter the device current. After incubation for 20 minutes, the 


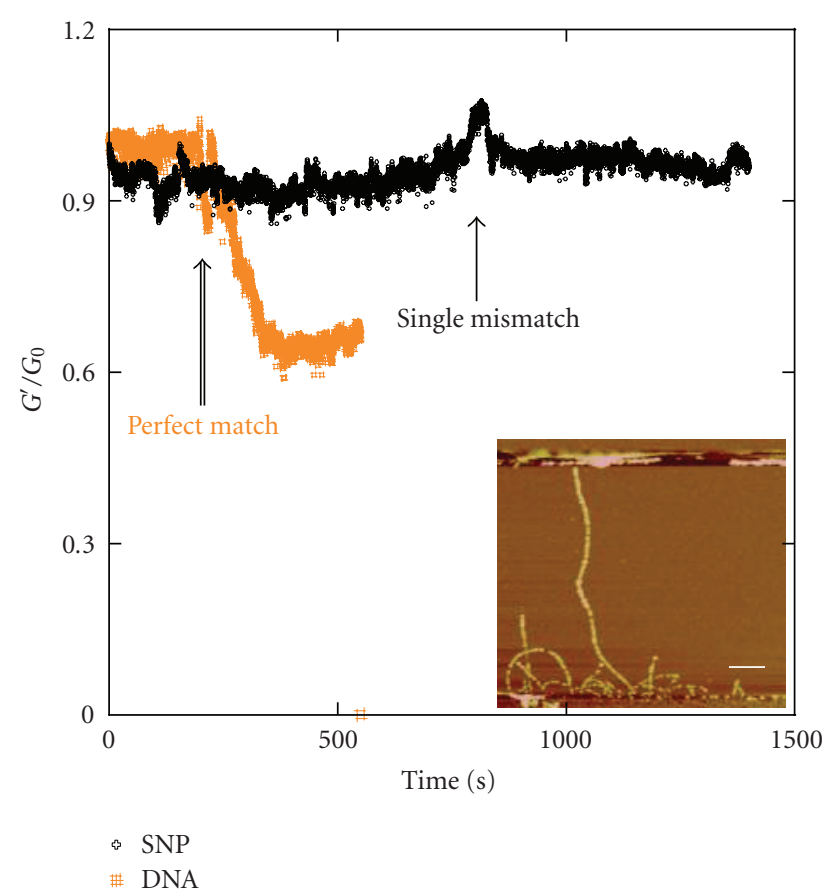

(a)

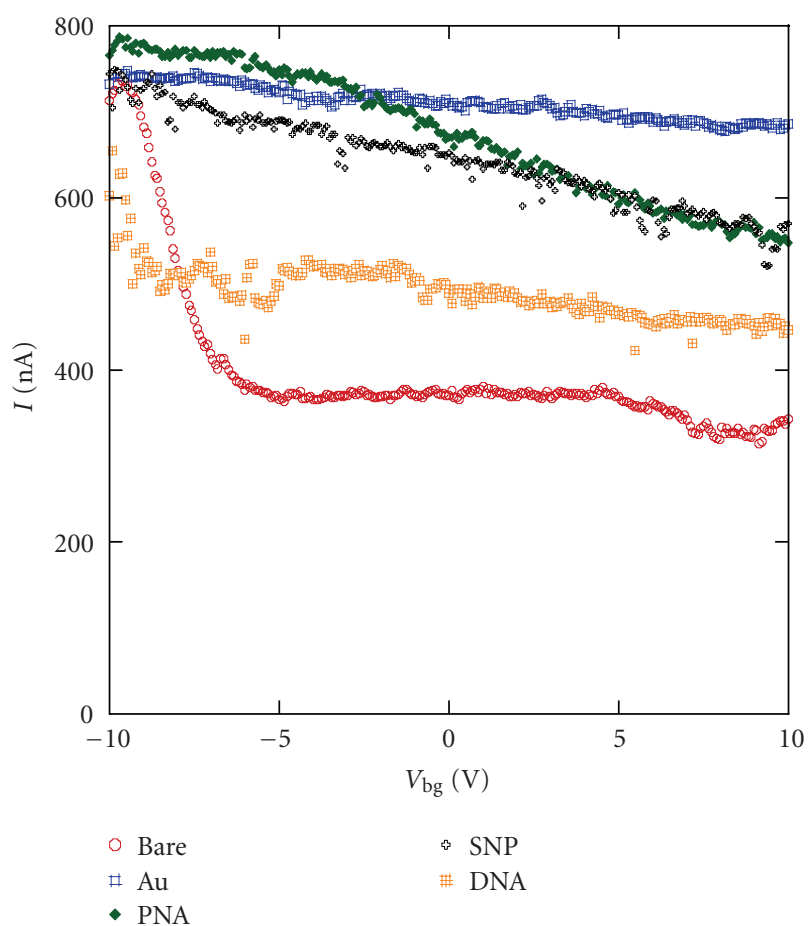

(b)

Figure 4: The effect of a single base mismatch. (a) Real-time conductance change upon adding target DNA with an SNP (black curve) and target ssDNA with perfect match (orange). Inset shows an AFM image of the device used for the measurement. Scale bar; $1 \mu \mathrm{m}$. (b) $I-V_{g}$ characteristics of the device at each functionalization and hybridization step. device was washed with an ample amount of DI water and blown dry with dry $\mathrm{N}_{2}$ gas. $I-V_{g}$ measurements taken after this did not show large changes either (Figure 4(b), black curve). Then, target ssDNA with perfect match was introduced to the same device baring $5 \mu \mathrm{L}$ DI water. As shown in Figure 4(a), clear decrease of conductance was observed upon introduction of matching DNA. Since we used the same concentration of target DNA in both SNP targets and matching targets $(100 \mathrm{pmole} / \mu \mathrm{L})$, we may assume that observed change of conductance is not due to the change of ionic conditions or change of $\mathrm{pH} . I-V_{g}$ measurement taken after washing and drying shows a clear decrease of conductance as well (Figure 4(b), orange curve).

We next employed a hybrid buffer to improve hybridization between probe PNA and target DNA sequence with an SNP to determine whether a single mismatch could really alter electrical characteristics. The hybrid buffer was $3 \mathrm{M} \mathrm{NaCl}, 10 \mathrm{mM}$ ethylenediaminetetraacetic acid (EDTA), and $380 \mathrm{mM}$ 4-(2-hydroxyethyl)-1-piperazineethanesulfonic acid (HEPES). Target DNA oligomers in the hybridization buffer were allowed to react with PNAs immobilized on Audecorated SWNT-FETs for 20 minutes. The chip was then washed with an ample amount of DI water to eliminate nonspecific binding and blown dry with $\mathrm{N}_{2}$ prior to further electrical characterization. We did not measure realtime changes in conductance as the high salt concentration of hybrid buffer made such assessment impossible. Figure 5(b) shows $I-V_{g}$ characteristics of the device before and after hybridization. In contrast to the absence of $I-V_{g}$ characteristic changes after hybridization in DI water, a clear decrease of conductance was observed after reaction in the hybrid buffer, as seen when sequences matched perfectly.

We thus conclude that the observed single-base mismatch discrimination shown by our device is likely not attributable to an SNP charge effect, as is the case in strain sensors $[35,36]$, but rather because hybridization of the SNP target is more difficult in DI water. To verify this, we labeled target SNP-bearing ssDNA with $\mathrm{Cy}-3$ and hybridized this material to PNAs immobilized on Au-decorated SWNTFETs in DI water for 20 minutes. No fluorescent signal was seen, in contrast to the result when perfectly matched target DNA was used for hybridization (data not shown). Park et al. have shown that ionic strength can affect PNADNA surface hybridization, although the influence of ionic strength is not as great as seen with DNA-DNA hybridization [37]. As we expect that probe PNAs are fully packed on Au nanoparticles, hybridizing sequences can communicate electrostatically with their neighbors, thereby hindering the hybridization of mismatched sequences.

In summary, we combined electrical transport in Audecorated SWNT-FET with confocal microscopy to measure hybridization of target ssDNA with PNAs. Uniform-sized Au nanoparticles were formed on sidewalls of SWNTs by electrochemical deposition, and the effect of Au-decoration appears as hole doping in most cases. By addition of thiolated PNA probes to such a device, we achieved stable immobilization of molecular recognition elements with minimal effects on electrical properties. Hybridization of complementary DNA 


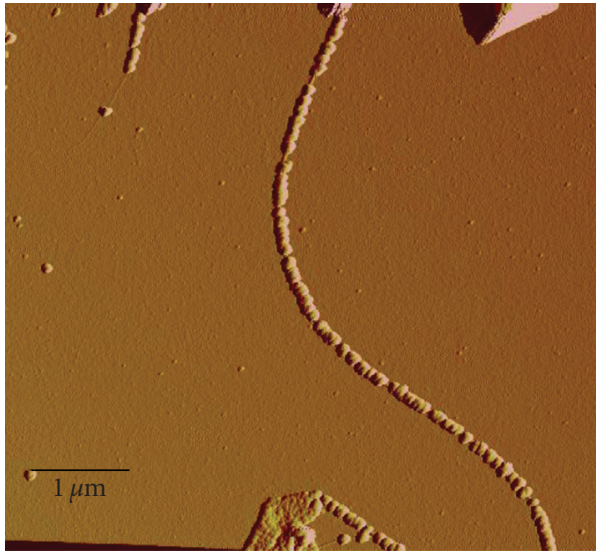

(a)

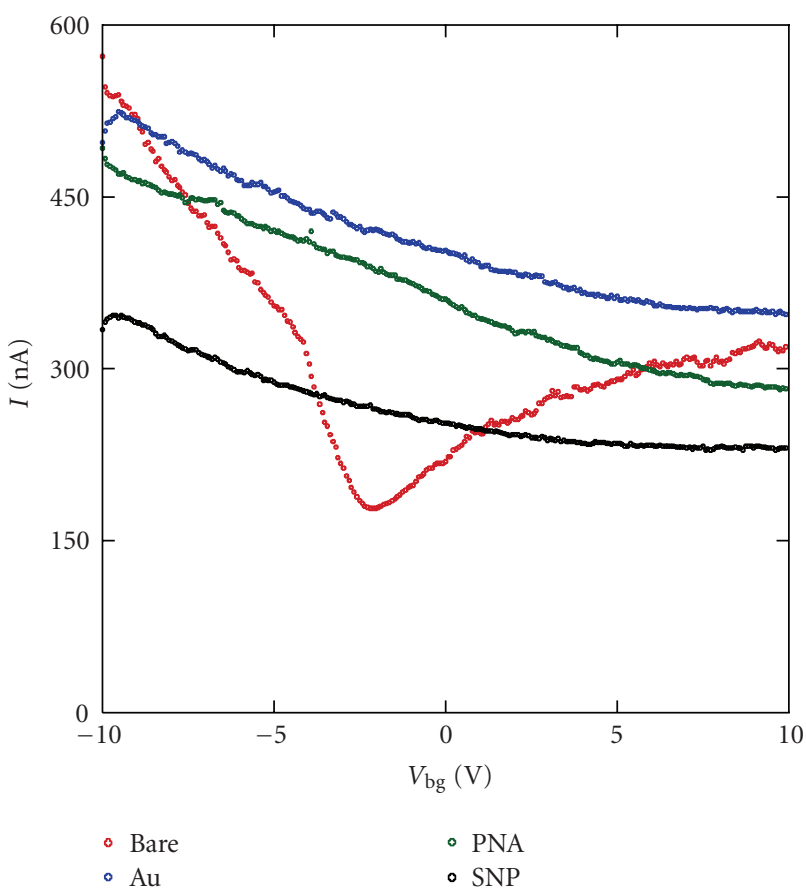

(b)

Figure 5: The effect of ionic concentration on target DNA hybridization when a single mismatch was present. (a) Atomic force micrograph of the Au-decorated device; (b) $I-V_{g}$ characteristics of the device at each functionalization and hybridization step.

in DI water resulted in a decrease in real-time conductance, and we confirmed that hybridization has indeed occured on Au nanoparticles by measuring fluorescence signal from Cy-3-labeled target DNA. In contrast, introduction of SNP target yields negligible change of conductance is in DI water. However, clear decrease of conductance observed when hybridization with SNP target is performed in hybridization buffer; SNP target is indiscernible from perfect match in high ionic conditions. Therefore, we may conclude that hybridization of mismatched DNA with PNA immobilized on nanoscale surfaces is difficult in low ionic conditions, at least in the timescale of 20 minutes.

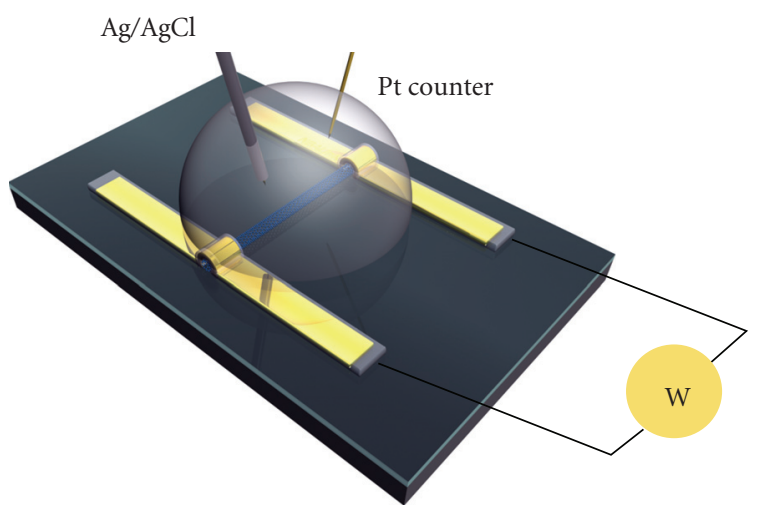

Scheme 1: Schematic diagram for Au decoration of SWNT-FETs.

\section{Experiments}

3.1. Device Fabrication. SWNTs were grown on a $\mathrm{Si} / \mathrm{SiO}_{2}$ substrate using a patterned chemical vapor deposition (CVD). Liquid catalyst consists of $\mathrm{Fe}\left(\mathrm{NO}_{3}\right)_{2} \cdot 9 \mathrm{H}_{2} \mathrm{O}$, and $\mathrm{Mo}(\mathrm{acac})_{2}$ in methanol was dispersed on substrates with patterned poly(methyl mettacrylate). After liftoff, SWNT growth was carried out in $900^{\circ} \mathrm{C}$-heated furnace for 10 minutes with $\mathrm{CH}_{4}$ or ethanol as a carbon source. To grow ultralong SWNTs, $0.5 \mathrm{M} \mathrm{FeCl}_{3}$ in DI water was placed at the edge of the sample. Ethanol was used as a carbon source, and the growth was carried out at $970^{\circ} \mathrm{C}$ for $20 \sim 30$ minutes. Patterns for electrical leads were formed by photolithography, followed by thermal evaporation of Ti and Au. For real-time characterization and metal decoration, all electrodes were insulated with $\mathrm{SiO}_{x}$ or SU8-2000.5 negative photoresist.

3.2. Au Decoration. By cyclic voltammetry or chronoamperometry, it is possible to control both Au nanoparticle size and packing density. Scheme 1 shows a schematic diagram of our Au-decoration process. A droplet of $100 \mu \mathrm{M} \mathrm{HAuCl}_{4}$ in $100 \mathrm{mM} \mathrm{KCl}$ (supporting electrolyte) was placed on the device, both source and drain electrodes were used as working electrodes, and a reduction potential was applied with respect to the $\mathrm{Ag} / \mathrm{AgCl}$ reference electrode with current monitoring using a Pt counter electrode. We found that cyclic voltammetry afforded better decoration control than did chronoamperometry. Uniform 20- to 60-nm-sized Au nanoparticles were formed on SWNTs by sweeping the potential from $-0.2 \mathrm{~V}$ to $0.6 \mathrm{~V}$.

\section{Supporting Information Available}

Optical microscope images and AFM images of Au-decorated $\mathrm{CNT}$ and electrical transport that show the effect of Au decoration, confocal micrographs showing $\mathrm{Cy}$-3-labeled probe PNA immobilization condition, both a confocal micrograph and a scanning electron micrograph of Au-decorated ultralong nanotubes after Cy3-labeled DNA hybridization, Electronic transfer characteristics of 5 different devices upon hybridization of DNA, and statistic of SWNT-FETs with nonspecifically bound PNA after the hybridization of matching DNA. 


\section{Acknowledgments}

Financial support was obtained from the Korea Research Council for Industrial Science and Technology, and Ministry of Environment as "The Eco-technopia 21 project." The authors thank Professor Hee Chul Choi and his group members for valuable discussion and assistance during this work and Professor Jing Kong's group for their help in growing ultralong nanotubes.

\section{References}

[1] S. J. Welsh and G. Powis, "Toward personalized theraphy for cancer," in Current Clinical Oncology: Targeted Cancer Theraphy, R. Kurzrock and M. Markman, Eds., pp. 411-412, Humana Press, Totowa, NJ, USA, 2008.

[2] F. Patolsky, A. Lichtenstein, and I. Willner, "Detection of single-base DNA mutations by enzyme-amplified electronic transduction," Nature Biotechnology, vol. 19, no. 3, pp. 253257, 2001.

[3] F. Pouthas, C. Gentil, D. Côte, and U. Bockelmann, "DNA detection on transistor arrays following mutation-specific enzymatic amplification," Applied Physics Letters, vol. 84, no. 9, pp. 1594-1596, 2004.

[4] Y. Xiao, X. Qu, K. W. Plaxco, and A. J. Heeger, "Label-free electrochemical detection of DNA in blood serum via targetinduced resolution of an electrode-bound DNA pseudoknot," Journal of the American Chemical Society, vol. 129, no. 39, pp. 11896-11897, 2007.

[5] G. Liu, Y. Wan, V. Gau et al., "An enzyme-based E-DNA sensor for sequence-specific detection of femtomolar DNA targets," Journal of the American Chemical Society, vol. 130, no. 21, pp. 6820-6825, 2008.

[6] Z. Li, Y. Chen, X. Li, T. I. Kamins, K. Nauka, and R. S. Williams, "Sequence-specific label-free DNA sensors based on silicon nanowires," Nano Letters, vol. 4, no. 2, pp. 245-247, 2004.

[7] J.-I. Hahm and C. M. Lieber, "Direct ultrasensitive electrical detection of DNA and DNA sequence variations using nanowire nanosensors," Nano Letters, vol. 4, no. 1, pp. 51-54, 2004.

[8] A. Star, E. Tu, J. Niemann, J.-C. P. Gabriel, C. S. Joiner, and C. Valcke, "Label-free detection of DNA hybridization using carbon nanotube network field-effect transistors," Proceedings of the National Academy of Sciences of the United States of America, vol. 103, no. 4, pp. 921-926, 2006.

[9] K. Besteman, J.-O. Lee, F. G. M. Wiertz, H. A. Heering, and C. Dekker, "Enzyme-coated carbon nanotubes as singlemolecule biosensors," Nano Letters, vol. 3, no. 6, pp. 727-730, 2003.

[10] H.-M. So, K. Won, Y. H. Kim et al., "Single-walled carbon nanotube biosensors using aptamers as molecular recognition elements," Journal of the American Chemical Society, vol. 127, no. 34, pp. 11906-11907, 2005.

[11] M. Zheng, A. Jagota, E. D. Semke et al., "DNA-assisted dispersion and separation of carbon nanotubes," Nature Materials, vol. 2, no. 5, pp. 338-342, 2003.

[12] W. Zhao, Y. Gao, M. A. Brook, and Y. Li, "Wrapping singlewalled carbon nanotubes with long single-stranded DNA molecules produced by rolling circle amplification," Chemical Communications, no. 34, pp. 3582-3584, 2006.
[13] E. S. Jeng, A. E. Moll, A. C. Roy, J. B. Gastala, and M. S. Strano, "Detection of DNA hybridization using the near-infrared band-gap fluorescence of single-walled carbon nanotubes," Nano Letters, vol. 6, no. 3, pp. 371-375, 2006.

[14] B.-K. Kim, N. Park, P. S. Na et al., "The effect of metal cluster coatings on carbon nanotubes," Nanotechnology, vol. 17, no. 2, pp. 496-500, 2006.

[15] D. R. Kauffman and A. Star, "Chemically induced potential barriers at the carbon nanotube-metal nanoparticle interface," Nano Letters, vol. 7, no. 7, pp. 1863-1868, 2007.

[16] H. R. Byon and H. C. Choi, "Network single-walled carbon nanotube-field effect transistors (SWNT-FETs) with increased schottky contact area for highly sensitive biosensor applications," Journal of the American Chemical Society, vol. 128, no. 7, pp. 2188-2189, 2006.

[17] V. Georgakilas, D. Gournis, V. Tzitzios, L. Pasquato, D. M. Guldi, and M. Prato, "Decorating carbon nanotubes with metal or semiconductor nanoparticles," Journal of Materials Chemistry, vol. 17, no. 26, pp. 2679-2694, 2007.

[18] H. C. Choi, M. Shim, S. Bangsaruntip, and H. Dai, "Spontaneous reduction of metal ions on the sidewalls of carbon nanotubes," Journal of the American Chemical Society, vol. 124, no. 31, pp. 9058-9059, 2002.

[19] B. M. Quinn, C. Dekker, and S. G. Lemay, "Electrodeposition of noble metal nanoparticles on carbon nanotubes," Journal of the American Chemical Society, vol. 127, no. 17, pp. 6146-6147, 2005.

[20] B. M. Quinn and S. G. Lemay, "Single-walled carbon nanotubes as templates and interconnects for nanoelectrodes," Advanced Materials, vol. 18, no. 7, pp. 855-859, 2006.

[21] Y.-S. Lo, D. H. Nam, H.-M. So et al., "Oriented immobilization of antibody fragments on ni-decorated single-walled carbon nanotube devices," ACS Nano, vol. 3, no. 11, pp. 3649-3655, 2009.

[22] D. S. Kim, T. Lee, and K. E. Geckeler, "Hole-doped singlewalled carbon nanotubes: ornamenting with gold nanoparticles in water," Angewandte Chemie, vol. 45, no. 1, pp. 104-107, 2005.

[23] S. Huang and Y. Chen, "Ultrasensitive fluorescence detection of single protein molecules manipulated electrically on $\mathrm{Au}$ nanowire," Nano Letters, vol. 8, no. 9, pp. 2829-2833, 2008.

[24] J. Zhang, Y. Fu, M. H. Chowdhury, and J. R. Lakowicz, "Metal-enhanced single-molecule fluorescence on silver particle monomer and dimer: coupling effect between metal particles," Nano Letters, vol. 7, no. 7, pp. 2101-2107, 2007.

[25] S. Shakeel, S. Karim, and A. Ali, "Peptide nucleic acid (PNA) a review," Journal of Chemical Technology and Biotechnology, vol. 81, no. 6, pp. 892-899, 2006.

[26] J. Wang, "DNA biosensors based on peptide nucleic acid (PNA) recognition layers. A review," Biosensors and Bioelectronics, vol. 13, no. 7-8, pp. 757-762, 1998.

[27] I. Y. Wong and N. A. Melosh, "Directed hybridization and melting of DNA linkers using counterion-screened electric fields," Nano Letters, vol. 9, no. 10, pp. 3521-3526, 2009.

[28] P. C. Rusu and G. Brocks, "Surface dipoles and work functions of alkylthiolates and fluorinated alkylthiolates on $\mathrm{Au}(111)$," Journal of Physical Chemistry B, vol. 110, no. 45, pp. 2262822634, 2006.

[29] B.-K. Kim, J.-J. Kim, H.-M. So et al., "Carbon nanotube diode fabricated by contact engineering with self-assembled molecules," Applied Physics Letters, vol. 89, no. 24, Article ID 243115, 2006. 
[30] I. Heller, A. M. Janssens, J. Männik, E. D. Minot, S. G. Lemay, and C. Dekker, "Identifying the mechanism of biosensing with carbon nanotube transistors," Nano Letters, vol. 8, no. 2, pp. 591-595, 2008.

[31] D. C. Hansen, K. M. Hansen, T. L. Ferrell, and T. Thundat, "Discerning biomolecular interactions using Kelvin probe technology," Langmuir, vol. 19, no. 18, pp. 7514-7520, 2003.

[32] X. Tang, S. Bansaruntip, N. Nakayama, E. Yenilmez, Y.-I. Chang, and Q. Wang, "Carbon nanotube DNA sensor and sensing mechanism," Nano Letters, vol. 6, no. 8, pp. 16321636, 2006.

[33] E. L. Gui, L.-J. Li, K. Zhang et al., "DNA sensing by field-effect transistors based on networks of carbon nanotubes," Journal of the American Chemical Society, vol. 129, no. 46, pp. 1442714432, 2007.

[34] A. Reina, M. Hofmann, D. Zhu, and J. Kong, "Growth mechanism of long and horizontally aligned carbon nanotubes by chemical vapor deposition," Journal of Physical Chemistry C, vol. 111, no. 20, pp. 7292-7297, 2007.

[35] J. Mertens, C. Rogero, M. Calleja et al., "Label-free detection of DNA hybridization based on hydration-induced tension in nucleic acid films," Nature Nanotechnology, vol. 3, no. 5, pp. 301-307, 2008.

[36] M. Cha, J. Shin, J.-H. Kim et al., "Biomolecular detection with a thin membrane transducer," Lab on a Chip, vol. 8, no. 6, pp. 932-937, 2008.

[37] H. Park, A. Germini, S. Sforza, R. Corradini, R. Marchelli, and W. Knoll, "Effect of ionic strength on PNA-DNA hybridization on surfaces and in solution," Biointerphases, vol. 2, no. 2, pp. 80-88, 2007. 

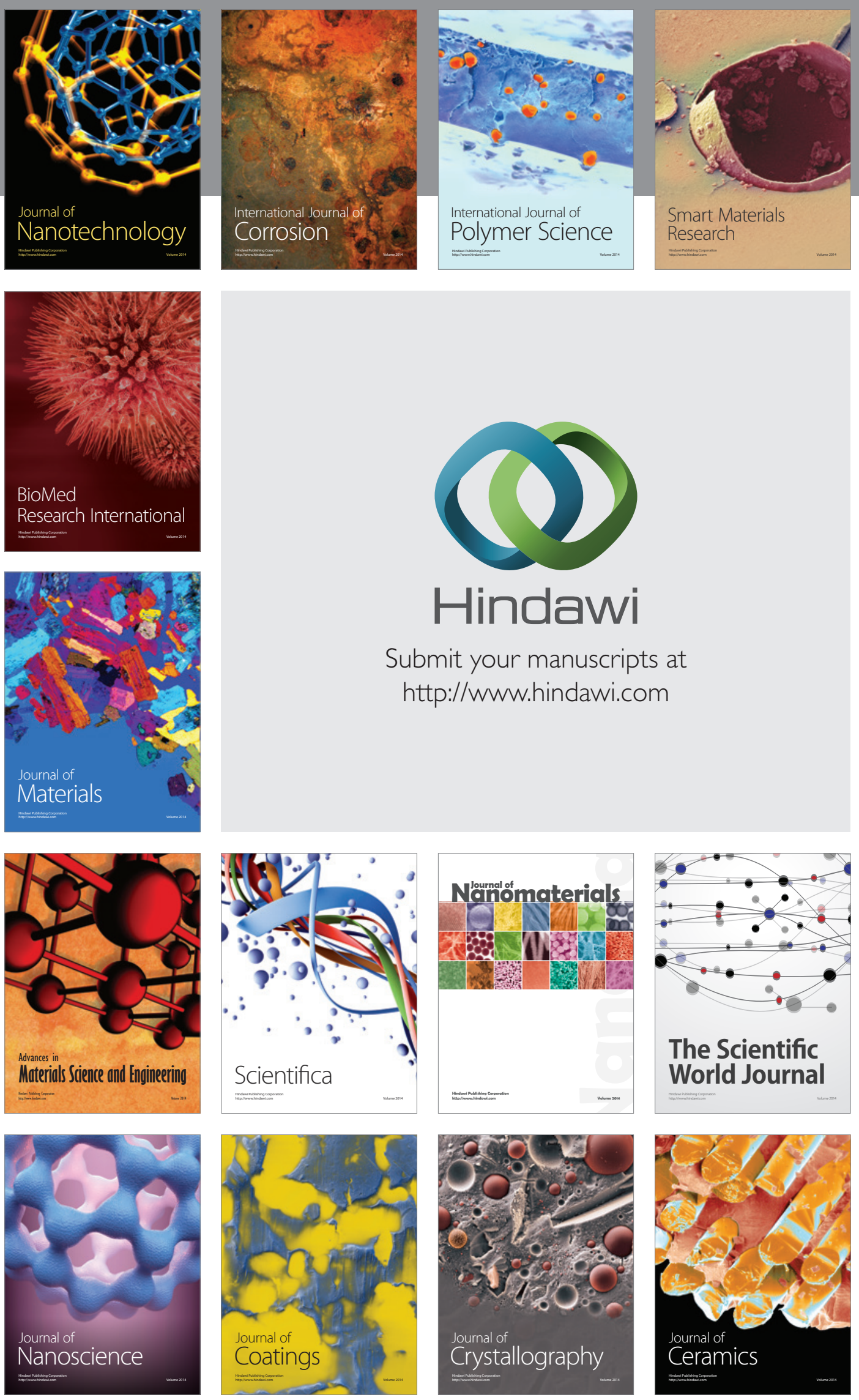

The Scientific World Journal

Submit your manuscripts at

http://www.hindawi.com

\section{World Journal}

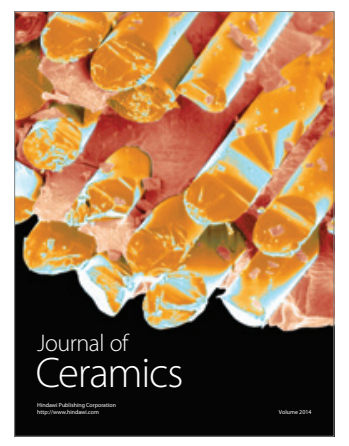

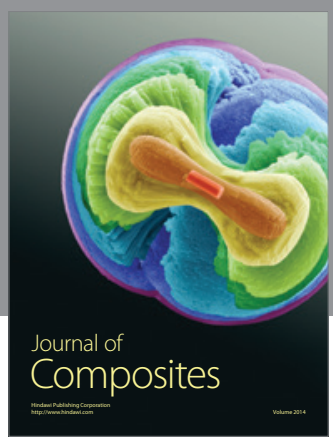
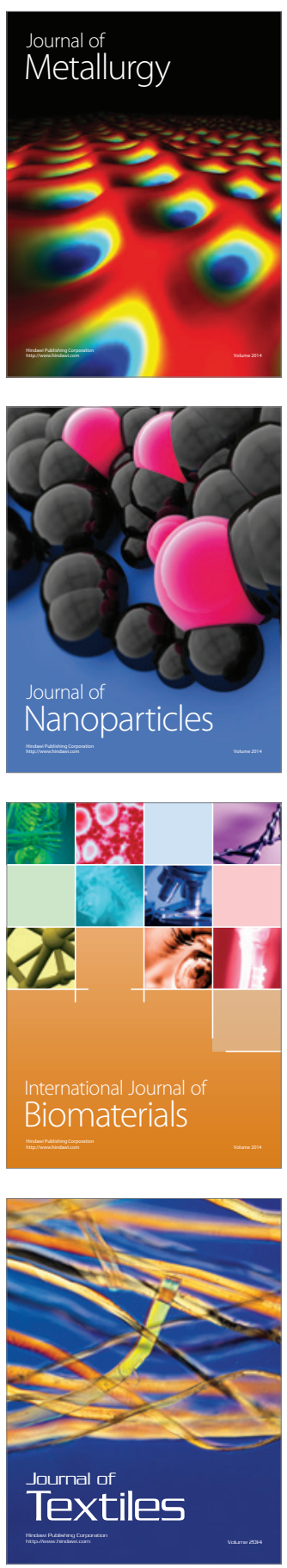